\begin{tabular}{|c|c|c|}
\hline & Int.J.Curr.Microbiol.App.Sci (2016) 5(12): $948-952$ & \multirow{2}{*}{ 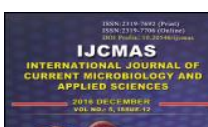 } \\
\hline & International Journal of Current Microbiology and Applied Sciences & \\
\hline & ISSN: 2319-7706 Volume 5 Number 12 (2016) pp. $948-952$ & \\
\hline EXCELLENT & Journal homepage: http://www.ijcmas.com & \\
\hline PUBLISHERS & & wwwijicmas com \\
\hline
\end{tabular}

Original Research Article

http://dx.doi.org/10.20546/ijcmas.2016.512.103

\title{
Variant Presentations of Mycetoma among Immunocompetent Patients attending a Tertiary Care Center in North Karnataka, India
}

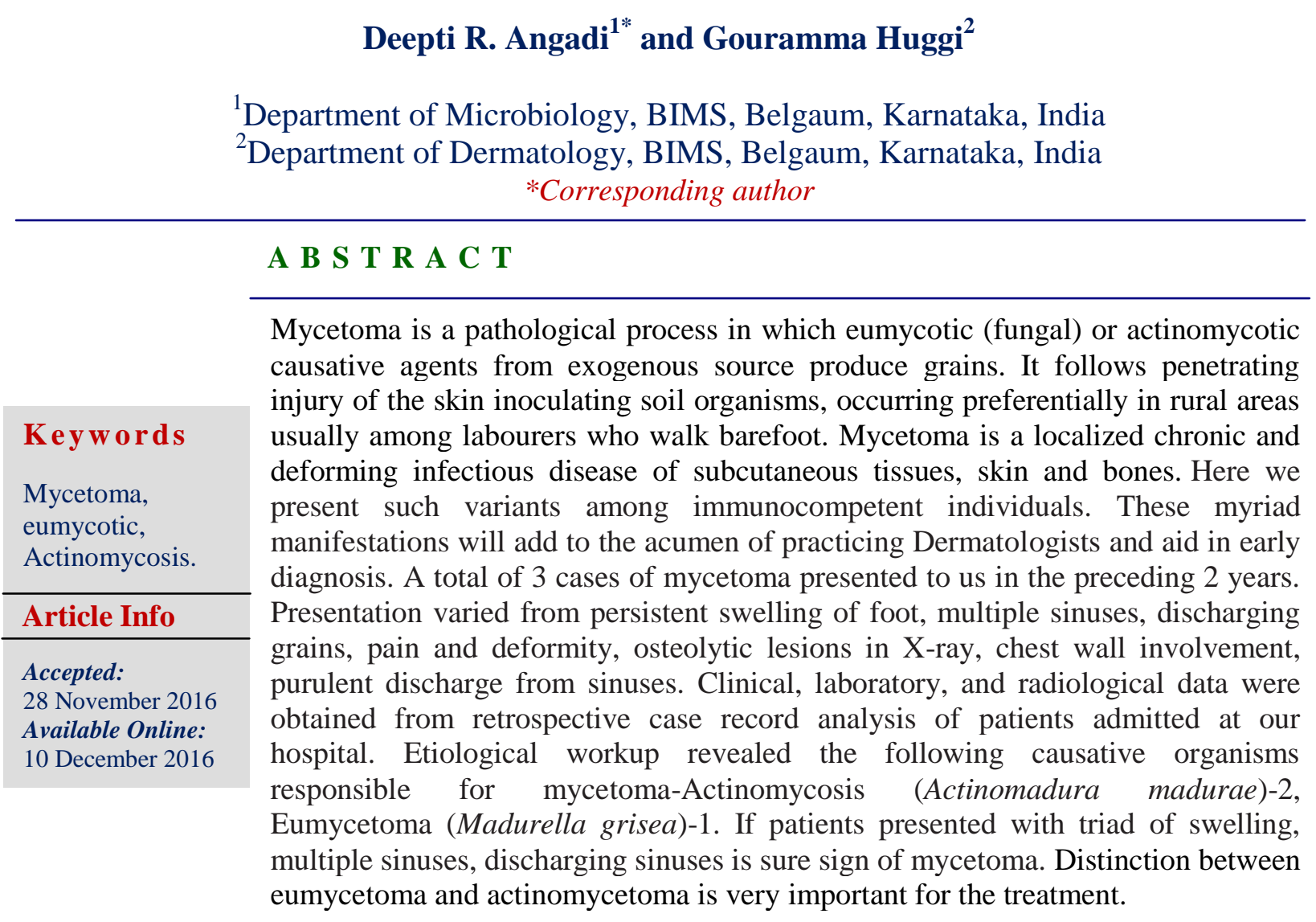

\section{Introduction}

Mycetoma is an uncommon condition that can affect any part of the body including the foot. It is more common in warm tropical and subtropical countries where people walk bare footed. Mycetoma is a pathological process in which eumycotic (fungal) or actinomycotic causative agents from exogenous source produce grains. It follows penetrating injury of the skin inoculating soil organisms, occurring preferentially in rural areas usually among labourers who walk barefoot.

Mycetoma is a localized chronic and deforming infectious disease of subcutaneous tissues, skin and bones (Zaias et al., 1969). The lesions are composed of suppurating abscesses and draining sinuses with the presence of grains which are characteristic of etiologic agents. In India, actinomycoticmycetoma is more commonly encountered than eumycoticmycetoma. However, the latter accounts for most cases reported from Northern India (Venugopal et al., 1994). 


\section{Case Reports}

Case 1: A 60 - year-old man, a farmer presented with a history of swelling of the right leg of 2 years duration with multiple discharging sinuses. He had a history of thorn prick one year prior to the onset of the lesion. On examination a large swelling measuring 14 x $10 \mathrm{~cm}$ seen in [Figure - 1]. It was firm in consistency. Multiple sinuses were seen with some of them discharging black granules. $\mathrm{KOH}$ examination from the granules revealed hyphae. Histopathological examination of the lesion was consistent with eumycaticmycetoma (Madurellagrisea). Radiological examination of the right leg revealed osteolytic lesion. The patient was treated with oral itraconozone and surgical management.

Case 2: A 32-year-old male laborer presented with swelling and multiple discharging sinuses over right ankle of 2 years. A painful nodule developed at the injury site, which he frequently manipulated with thorns and needles; and progressively increasing painful swelling of the ankle. Clinical examination showed diffuse, illdefined, indurated swelling over lateral side of ankle, It was studded with multiple, mildly tender, soft nodules and a few sinuses having sero-purulent discharge. Systemic examination and routine investigations, including complete blood counts, serum biochemistry, urinalysis, and chest $\mathrm{x}$-ray films, did not show any abnormality.

Microscopy of sero-purulent material in potassium hydroxide $(\mathrm{KOH})$ mounts showed multilobulated, vermiform grains. Sabouraud. Dextrose agar (SDA) without antibiotics, and Lowenstein-Jensen media. Aerobic culture on blood agar grew characteristic chalky white colonies of Nocardiaspp. on the 4thday . Gram-stained smears from these colonies showed gram- positive, branching filamentous bacilli which were also weakly acid fast with modified Z-Nstain, indicative of Nocardiaspp. We are treated this patient with Welsh regimen.

Case 4: A 43 year male patient presented with swelling of left foot, multiple watery discharging wounds, and blackish discoloration of foot with pain. The patient was injured three years before the onset of the clinical symptoms. On examination swelling of Present over left foot up to ankle joint, hyper pigmentation of foot, multiple discharging sinuses with seropurulent discharge and black crusting. Systemic examination and all routine blood and urine analysis are normal. $\mathrm{KOH}$ fungal culture confirmed the diagnosis as actinomycosis.

\section{Results and Discussion}

Mycetoma as a medical entity was first reported as Madura foot in Madurai, India in 1842 (Rippon, 1982). Mycetoma (Madura foot, maduromycosis) is a chronic, localized infection of the dermis and subcutaneous tissue with indolent swelling and draining sinuses The granules in the discharge are actually aggregates of microcolonies of the organism. More than 20 species of fungi and bacteria have been implicated as etiologic agents of mycetoma. Approximately $40 \%$ of cases are due to true fungi (eumycetoma), and $60 \%$ are caused by aerobic actinomycetes (actinomycetoma).

Traumatic implantation or contamination of a wound involving the limb (foot) in majority of the cases (Papaioannides et al., 2001). Since the lesions are invariably triggered by injury, involvement of back, arm, leg or thigh is unusual and reported infrequently (Sharma et al., 1979; Annamalai et al., 1997). Upper back particularly gets involved in persons 
habitually carrying wood or soiled sacks on the back. Men/women 20 to 50 years of age, walking barefoot, and working outdoors in rural settings are particularly at risk.

Fig.1 Eumycotic mycetoma (Madurella grisea)

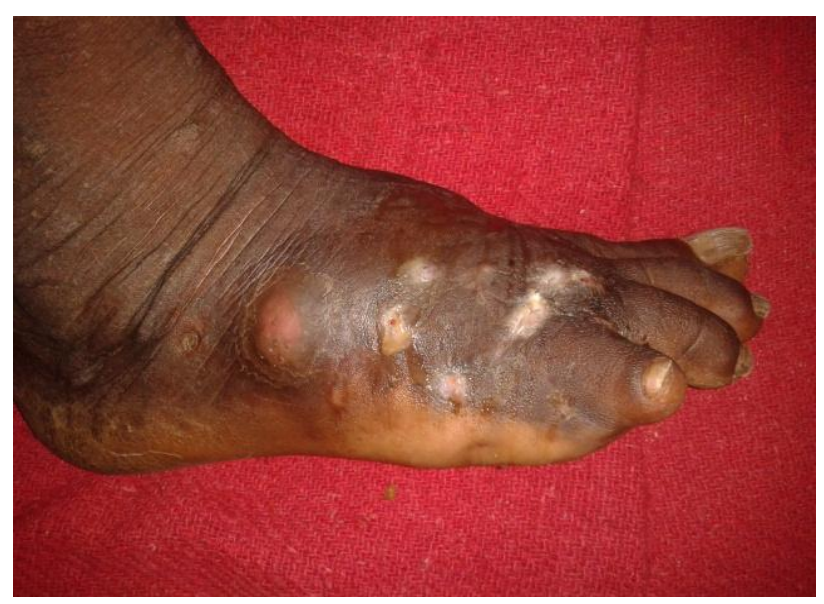

Fig.2 Actinomycotic mycetoma (Nocardia Species)

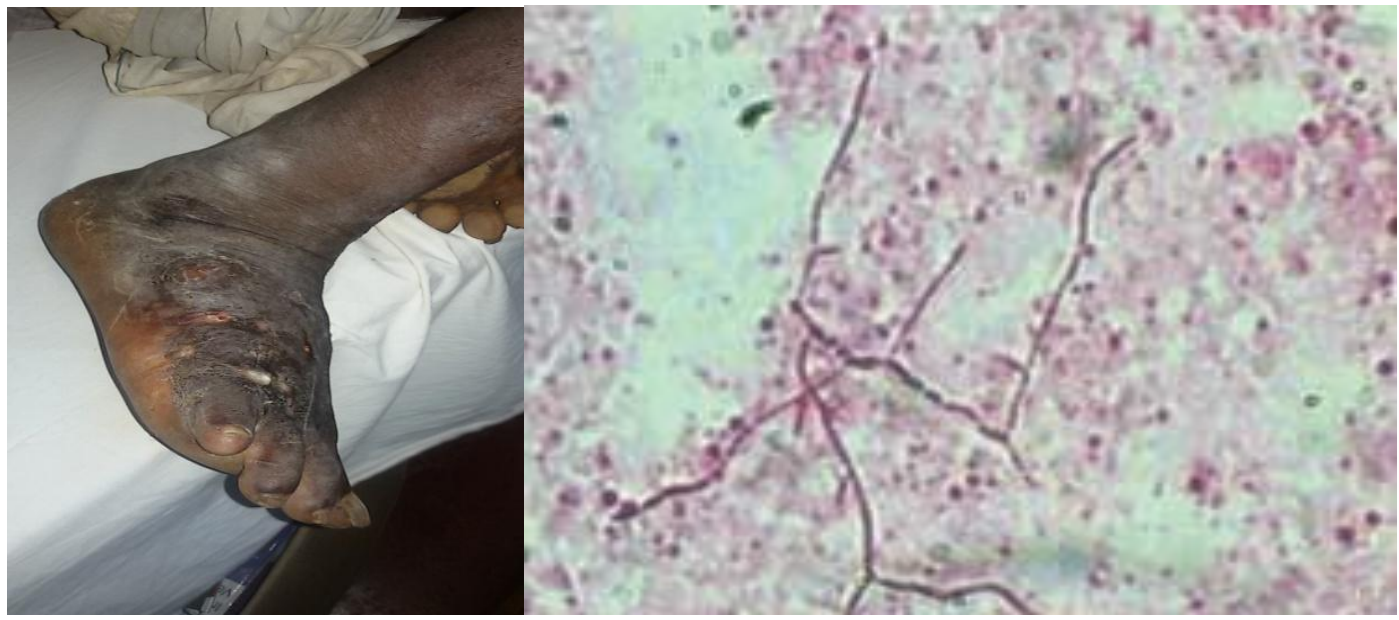

Fig.3 Actinomycotic mycetoma

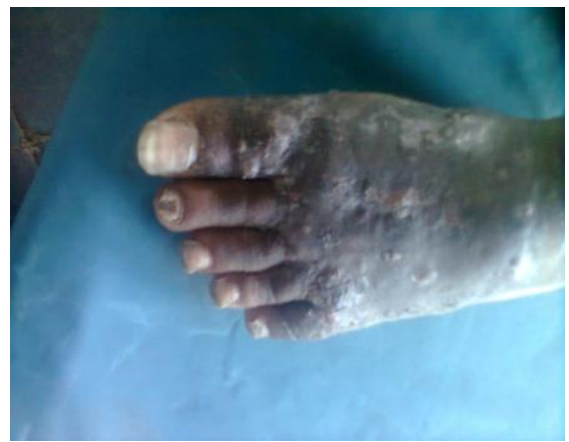


Progression to fibrosis, mutilation, and finally loss of function is rapid in actinomycotic/nocardial mycetoma cases. Bone involvement occurs infrequently. The notable bony changes include periosteal thickening, osteoporosis and osteolysis (Hazra et al., 1998). The treatment of choice is surgical excision with wide margins; antifungal or antibiotic treatment following surgical excision is debatable as the results are variable. Antifungal and antibiotic treatment can be used depending on the type of organism causing the lesion. For Actinomycetoma, combination therapy with trimethoprim-sulfamethoxazole, dapsone and streptomycin has been used. Rifampicin has been used in resistant cases (Ania et al., 2008). Azole treatment is the recommended regime for small Eumycetoma lesions in the extremities. Madurellamycetomatis may respond to ketoconazole and $P$. boydii $(S$. apiospermum) may respond to itraconazole. Other agents of Eumycetoma may respond intermittently to itraconazole or amphotericin B (Lexier et al., 1999). However, Agarwal and colleagues have recently described their experience of two different regimens of medical treatment for patients with actinomycetoma of the foot and observed that the Ramam regimen was found to be very effective in treating such patients with only minimal bony involvement, while the Welsh regimen and its modification are suitable for more severe cases, because amikacin is more sensitive than gentamicin in the treatment of resistant organisms. The modified Welsh regimen can also be continued for five cycles when there is more extensive bony involve men. The diagnosis in our patients was supported by $\mathrm{KOH}$ examination and confirmed by histo pathological examination.

In conclusion, if patients presented with triad of swelling, multiple sinuses, discharging sinuses is sure sign of mycetoma. Distinction between eumycetoma and actinomycetoma is very important for the treatment.

\section{References}

Agarwal, U.S., Besarwal, R.K., Gupta, R., Agarwal, P. 2012. Treatment of actinomycetoma foot - our experience with ten patients. J. Eur. Acad. Dermatol. Venereol.

Ahmed, A.A., van de Sande, W.W., Fahal, A., Bakker-Woudenberg, I., Verbrugh, H., van Belkum, A. 2007. Management of Mycetoma: major challenge in tropical mycoses with limited international recognition. Curr. Opin. Infect Dis., 20(2): 146-51.

Ania, B.J., Asejo, M., Kiel, R.J. 2008. Mycetoma Mycetoma, e Medicine.

Annamalai, R., Pankaja, V.V., Lakshmi, V.V., Pandurangan, C.N., Subramanian, S. 1997. Mycetoma in an unusual site. Indian J. Dermatol. Venereol. Leprol., 43: 324-7.

Chander, J. 2002. Mycetoma. In: Textbook of Medical Mycology. In: Chander J, editor. 1st ed. New Delhi: Mehta Publishers, p. 114-27.

Fahal, A.H., Hassan, M.A. 1992. Mycetoma. Br. J. Surg., 79(11): 1138-41.

Hazra, B., Bandyopadhyay, S., Saha, S.K., Banerjee, D.P., Dutta, G. 1998. A study of mycetoma in eastern India. $J$. Common Dis., 30: 7-11.

Lexier, R., Walmsley, S.L. Successful treatment of Madura foot caused by Pseudallescheriaboydii with Escherichia coli super infection: a case report. Can. J. Surg., 42(4): 307-9.

Papaioannides, D., Akritidis, N.K. 2001. Painless foot swelling with a chronic purulent discharge. West J. Med., 174: 96-7.

Rippon, J.W. 1982. Mycetoma In: Rippon, Medical Mycology. The pathogenic 
fungi and the pathogenic actinomycetes. 2 nd edition: WB Saunders, 79.

Sharma, S.C., Kumar, B., Talwar, P., Bedi, T.R., Kaur, $\quad$ S. 1979. Nocardialmycetoma at unusual site. Indian J. Dermatol. Venereol. Leperol., 45: 371-3.

Venugopal, P.V., Venugopal, T. 1994. Deep fungal infections In: Textbook and Atlas of Dermatology 1" edition, edited by Valia RG, Bhalani Publishing House, Bombay, 213.

Zaias, N., Taplin, D., Gerbert. 1969. Mycetoma. Arch. Dermatol., 99: 21525.

\section{How to cite this article:}

Deepti R. Angadi, Gouramma Huggi. 2016. Variant Presentations of Mycetoma among Immunocompetent Patients attending a Tertiary Care Center in North Karnataka, India. Int.J.Curr.Microbiol.App.Sci. 5(12): 948-952. doi: http://dx.doi.org/10.20546/ijcmas.2016.512.103 